\title{
Interaction between Genetic Effects and Soil Type in Diallel Analysis of Root Shape and Size of Japanese Radish (Raphanus sativus L.)
}

\author{
Hiroyoshi Iwata*1), Satoshi Niikura2), Seiji Matsuura'), Yasushi Takano ${ }^{3)}$ and Yasuo Ukai ${ }^{3)}$ \\ 1) Department of Information Science and Technology, National Agricultural Research Center, 3-1-1 Kannondai, Tsukuba, Ibaraki 305- \\ 8666, Japan \\ 2) Tohoku Seed Company, 1625 Nishihara, Himuro, Utsunomiya, Tochigi 321-3232, Japan \\ 3) Department of Agricultural-Environmental Biology, Graduate School of Agriculture and Life Sciences, The University of Tokyo, 1-1-1 \\ Yayoi, Bunkyo, Tokyo 113-8657, Japan
}

\begin{abstract}
We conducted diallel analyses of root shape and size in Japanese radish to determine the interaction between genetic effects and soil type in the selection for these traits in a breeding program. A set of diallel crosses of six inbred lines was grown in an andosol, a clayey soil and a sandy soil. The major shape characteristics accounted for by the principal components of elliptic Fourier descriptors were the ratio of length to width, the bluntness of the distal part, the curvature of the central part, and the curvature of the proximal and distal parts. Analysis of variance of diallel tables showed that the genetic effects were highly significant for all the characteristics. In contrast, the influence of the soil type was markedly different among the characteristics. Soil type effect was significant for size characteristics and the bluntness of the distal part. In the bluntness of the distal part, the interaction between genetic effects and soil type was significant, and the heritability and the degree of dominance varied between the soil types. The results indicated that the differences in the soil types did not influence the selection for the root size or shape in most characteristics. However, the interaction between genetic effects and soil type should be taken into account in the selection for the bluntness of the distal part.
\end{abstract}

Key Words: genotype by environment interaction, soil type, diallel analysis, radish root, shape and size, elliptic Fourier descriptors, image analysis.

\section{Introduction}

Radish is an important root vegetable, especially in East Asia. Storage root shape varies among Japanese cultivars of radish, which have been genetically improved to meet the demand from consumers (Nishiyama 1958). Cur-

Communicated by K. Takeda

Received December 1, 2003. Accepted June 7, 2004

The first two authors contributed equally to this work

*Corresponding author (e-mail: iwatah@affrc.go.jp) rently, what constitutes a desirable root shape has become more variable and subtle than before, because of the diversification of consumer requirements. The shape and size of root vegetables are determined not only by the genotype but also by environmental conditions, especially by soil types (e.g. Rosenfeld et al. 1998a, 1998b, 1999, Goldman 1995, Villordon and Carroll 2002). Information about the soil type effect and the interaction between genotype and soil type, as well as genotypic effect, is, therefore, indispensable to identify the optimum soil type in the selection for these characteristics and to select suitable parental lines efficiently.

We previously evaluated the radish root shape by using elliptic Fourier descriptors (EFDs) (Kuhl and Giardina 1982), and observed that independent shape characteristics can be measured quantitatively as scores of principal components of the EFDs (Iwata et al. 1998). In the previous study, we examined the effects of the genotype, soil type and growth stage, and also the interaction among these three factors in the variations of the root shape in radish. We reported that the root shape can be evaluated by the addition of these three main effects, because they did not show any significant interaction with each other in their effects on most shape characteristics. The previous study, however, was not based on data from diallel mating experiments, and could not provide information about the influence of the soil type on the mode of inheritance of the radish root shape. We have also conducted diallel analyses of the shape characteristics of radish roots to evaluate the mode of inheritance of the root shape and size (Iwata et al. 2000). Since this study was performed with a single soil type, it was not possible to determine the interaction between genetic effects and soil types.

In the present study, we conducted diallel analyses of the root shape and size in three different soil types. The objectives of the study were to evaluate any interactions between the soil type and genetic effects and to elucidate the differences in the mode of inheritance between soil types if interactions exist. We analyzed the influence of the soil type on the selection for the root size and shape of radish.

\section{Materials and Methods}

Plant materials and experimental design

Six inbred lines obtained after eight (line A), seven 
(lines B, C, D and E), or four (line F) generations of full-sib inter-crossing of the members of the 'Miyashige' cultivar group, whose members are generally used as parents of commercial $F_{1}$ varieties in Japan presently (Niikura and Matsuura 1999), were crossed in all possible combinations. Parents A, C and E display relatively thin, D thick, and B and $\mathrm{F}$ intermediate roots. The typical root shape of each parent is illustrated in Figure 1 in the report of Iwata et al. (2000), which represents typical variations in the 'Miyashige' cultivar group (Niikura and Matsuura 1999). Seeds of the six parental lines and the $30 \mathrm{~F}_{1}$ hybrids were sown on September 5, 1996 in three experimental fields, each with a different soil type (andosol, clayey soil and sandy soil), in Utsunomiya, Tochigi, Japan (lat. $36^{\circ} 31^{\prime} \mathrm{N}$, long. $139^{\circ} 59^{\prime} \mathrm{E}$ ). Fertilizer was applied before sowing at the rates of $1.4 \mathrm{~g} \mathrm{~N}, 1.7 \mathrm{~g} \mathrm{P}_{2} \mathrm{O}_{5}$ and $1.3 \mathrm{~g} \mathrm{~K}_{2} \mathrm{O}$ per $\mathrm{m}^{2}$. Plowing depth was $40 \mathrm{~cm}$. Each diallel set of plants was arranged in a randomized block design with two replications. Each block consisted of 36 plots. Plants were spaced $27 \mathrm{~cm}$ apart in ridges spaced $45 \mathrm{~cm}$ apart. Samples were harvested on November 5, 1996; five plants were sampled from each plot. The samples from the andosol field were identical with those reported previously (Iwata et al. 2000).

\section{Determination of root shape and size}

The method for quantifying the shape variations of the sampled roots was described previously in detail (Iwata et al. 1998, 2000). In summary, the root contour coordinates extracted by image analysis were described by 77 coefficients of normalized EFDs (Kuhl and Giardina 1982). The principal component analyses were conducted separately for two groups of coefficients, which were related to the symmetrical (group A) and asymmetrical (group B) shape variations with respect to the central axis of the root. The shape variation accounted for by each component was visualized by the method developed by Rohlf and Archie (1984). A series of analyses was performed by using the software package SHAPE (Iwata and Ukai 2002). We also measured the root size as the two-dimensional projection area of the root
(RA) based on image analysis and root weight (RW).

\section{Diallel analysis}

Before the analysis, the principal component scores and size characteristics were averaged over the five sample plants per plot. The scores of the principal components of group B coefficients were converted to absolute values before being averaged, in order to remove the influence of the direction of curvature of the root.

In order to evaluate the genetic effect, soil type effect, and the interaction between them, we incorporated the terms related to the soil type into Hayman's model for the analysis of variance (ANOVA) of diallel tables (Hayman 1954a). The significance of each item in the model was tested against the mean squares of pooled block interaction. The genetic parameters were estimated and graphically analyzed in relation to the model of Hayman (1954b). The broad- and narrow-sense heritabilities were estimated with the equations proposed by Mather and Jinks (1971). In the estimation and graphical analysis of genetic parameters, data were analyzed separately for each soil type, and error variance was estimated based on the interaction between block and genotype.

\section{Results}

The first two components of groups A and B accounted for over $95 \%$ of the total variance (Table 1). From the reconstructed contours, the first two components of groups A and $\mathrm{B}$ were found to reflect the type of shape variation similar to that observed in Figure 2 in the report of Iwata et al. (2000). That is, 1st and 2nd principal components of group A (AP1 and AP2) were adequate measures of the ratio of length to width and of the bluntness of the distal part of the root, respectively. The 1st and 2nd components of group B (BP1 and BP2) were associated with the curvature in the central part and the curvature in the proximal and distal parts, respectively.

ANOVA of diallel tables was performed across soil

Table 1. Eigenvalues and contributions of principal components of groups A and B coefficients of elliptic Fourier descriptors for 36 entries grown in three soil types. The coefficients in groups A and B were related to the symmetrical and asymmetrical shape variations with respect to the central axis of the root, respectively

\begin{tabular}{|c|c|c|c|c|c|c|}
\hline \multirow{3}{*}{ Component } & \multicolumn{3}{|c|}{ Group A } & \multicolumn{3}{|c|}{ Group B } \\
\hline & \multirow{2}{*}{ Eigenvalue $^{1)}$} & \multicolumn{2}{|c|}{ Proportion (\%) } & \multirow{2}{*}{ Eigenvalue $^{1)}$} & \multicolumn{2}{|c|}{ Proportion (\%) } \\
\hline & & Within group ${ }^{2)}$ & Total $^{3)}$ & & Within group ${ }^{2)}$ & Total $^{3)}$ \\
\hline 1 & 314.08 & 93.93 & 51.88 & 245.62 & 90.62 & 40.57 \\
\hline 2 & 9.06 & 2.71 & 1.50 & 12.64 & 4.66 & 2.09 \\
\hline 3 & 4.20 & 1.26 & 0.69 & 5.40 & 1.99 & 0.89 \\
\hline 4 & 1.82 & 0.54 & 0.30 & 3.06 & 1.13 & 0.51 \\
\hline 5 and lower & 5.21 & 1.56 & 0.86 & 4.34 & 1.60 & 0.72 \\
\hline Total & 334.37 & 100.00 & 55.23 & 271.06 & 100.00 & 44.77 \\
\hline
\end{tabular}

1) Eigenvalues are scaled by $10^{-5}$.

2) Proportion relative to the total variance in each group.

3) Proportion relative to the total variance in both groups. 
Table 2. Computation of $F$ ratios for ANOVA of diallel tables for the 1st and 2nd principal components of group A (AP1, AP2) and group B (BP1, BP2) coefficients, two-dimensional projection area (RA), and root weight (RW). See text for the meaning of each principal component

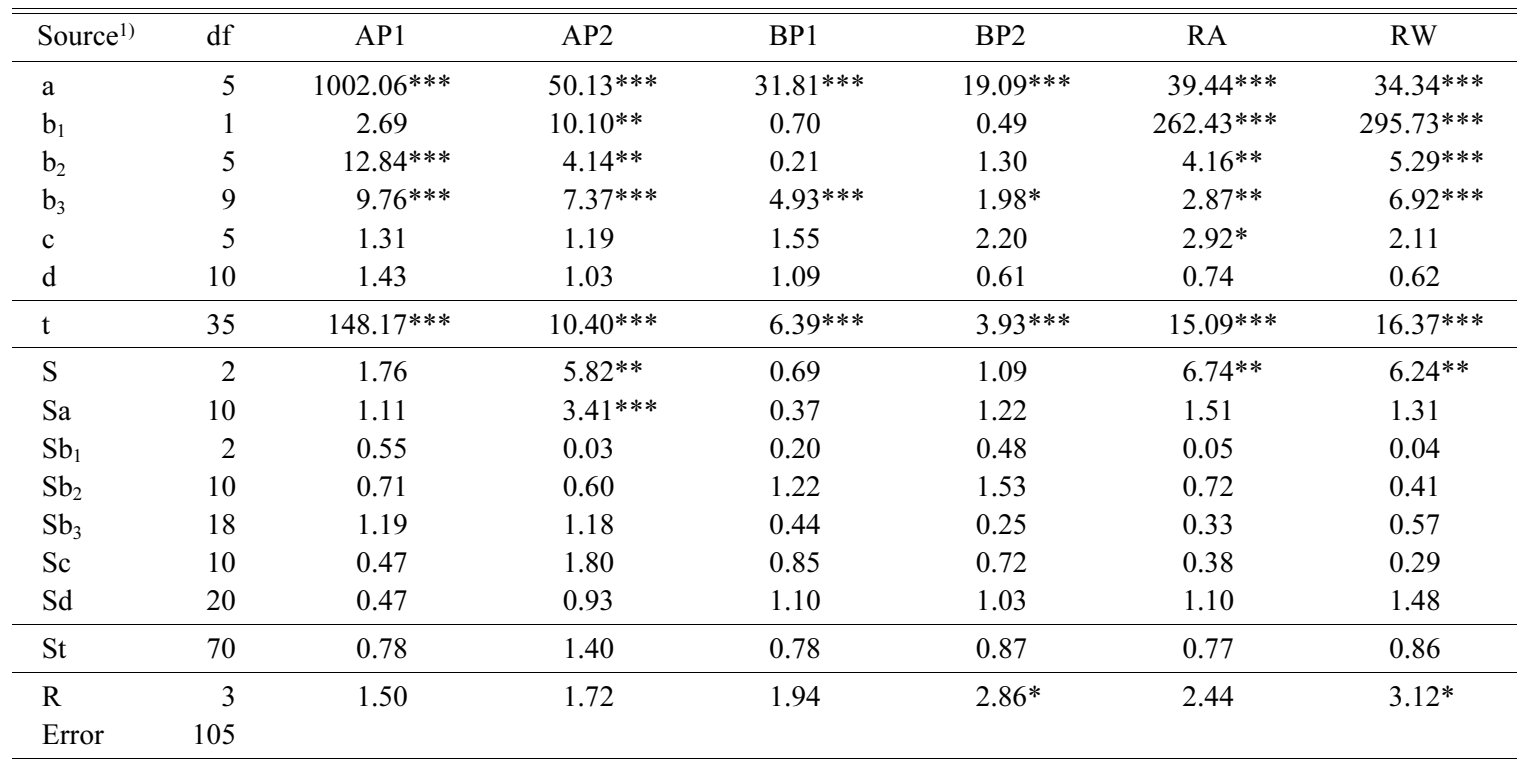

1) $a$, additive effect; $b_{1}$, mean dominance deviation; $b_{2}$, dominance deviation due to parents; $b_{3}$, residual dominance; $c$ : average maternal effects; $d$ : variation in reciprocal differences not ascribable to $c$; $t$, sum of genetic effects; $S$, effect of soil type; $\mathrm{Sa}-\mathrm{Sd}$, interactions between soil type and genetic effects; $\mathrm{St}$, the sum of interaction between soil type and genetic effects; R, block effect; Error, pooled block interactions.

$*, * *, * *$ : Significant at $P<0.05,0.01$, or 0.001 , respectively.

types for each of six characteristics (Table 2). The additive genetic effect (a) was significant at the 0.001 probability level for all the characteristics. The mean dominance deviation $\left(b_{1}\right)$ was significant for AP2 and size characteristics (RA and $\mathrm{RW}$ ), indicating that there is a directional dominance effect in these characteristics. The additional dominance deviation due to parents $\left(b_{2}\right)$ was significant except for asymmetric shape variations (BP1 and BP2). The significance of $b_{2}$ indicated that some parents harbored considerably more dominant alleles than others. The residual dominance $\left(b_{3}\right)$, which tests the part of the dominance deviation that is unique to each $F_{1}$, was significant for all the characteristics. The maternal effects, i.e., c and d, were not significant, except for c in RA. The sum of the genetic effects $(\mathrm{t})$ was significant at the 0.001 level for all the characteristics. The soil-type effect (S) was significant for AP2 and size characteristics at the
0.01 level. The interactions between genetic effects and soil type were significant only in one case; the interaction between additive effect and soil type (Sa) in AP2 at the 0.001 level. The sum of interactions between soil type and genotype (St) was not significant at the 0.05 level for all the characteristics, while it was marginally significant $(P<0.06)$ for AP2. Since the interaction between genetic effects and soil type was significant only for AP2, the results of the following analyses are presented only for this characteristic.

The genetic parameters varied among the soil types (Table 3). The broad- and narrow-sense heritabilities were highest in the andosol. In the $\left(\mathrm{V}_{\mathrm{r}}, \mathrm{W}_{\mathrm{r}}\right)$ analysis, the regression line with unit slope intersected the $\mathrm{W}_{\mathrm{r}}$-axis above the origin in the andosol and sandy soil and at the origin in the clayey soil, indicating the presence of incomplete and complete dominance, respectively (Fig. 1). Parents B, C and D

Table 3. Genetic parameters estimated for 2 nd principal component of the group A coefficients (AP2) in each soil type

\begin{tabular}{|c|c|c|c|}
\hline & Andosol & Clayey soil & Sandy soil \\
\hline Average degree of dominance ${ }^{1)}$ & 0.617 & 0.991 & 0.526 \\
\hline Proportion of dominant genes ${ }^{1)}$ & 0.707 & 0.520 & 0.567 \\
\hline Mean direction of dominance $(\text { Sign of } h)^{1)}$ & + & + & + \\
\hline Correlation between $\mathrm{V}_{\mathrm{r}}+\mathrm{W}_{\mathrm{r}}$ and $\left.\mathrm{P}_{\mathrm{r}}{ }^{1}\right)$ & -0.616 & $-0.898 *$ & -0.051 \\
\hline Heritability $(\text { Broad })^{2)}$ & 0.855 & 0.804 & 0.707 \\
\hline Heritability (Narrow) ${ }^{2)}$ & 0.700 & 0.554 & 0.580 \\
\hline
\end{tabular}


showed large differences in location on the $\left(\mathrm{V}_{\mathrm{r}}, \mathrm{W}_{\mathrm{r}}\right)$ graphs between the different soil types compared with the parents A, E and F. For example, parent B was located near the origin in the andosol and sandy soil, indicating that the alleles carried by B were dominant in these soil types. Mean direction of dominance, i.e., the sign of $\mathrm{h}$ (Hayman 1954b), was positive, indicating that most of the alleles for the bluntshaped roots were dominant in all the soil types (Table 3 ). This fact was also indicated by the negative correlation between the degree of dominance of the parents $\left(\mathrm{W}_{\mathrm{r}}+\mathrm{V}_{\mathrm{r}}\right)$ and the parental value $\left(\mathrm{P}_{\mathrm{r}}\right)$, although the correlation was significant only for the clayey soil.

\section{Discussion}

We evaluated quantitatively the variations in root shape of radish by using the scores of principal components of EFDs. The reproducibility and stability of the principal components of EFDs should be confirmed when the component scores are used as key characteristics for evaluating variations in the root shape of radish. Through visual evaluation of the reconstructed contours, we found that the first four components reflected similar types of shape variations to those observed in our previous studies (Iwata et al. 1998, 2000). This suggests that the scores of these components can certainly be used as key characteristics.

In the diallel analysis according to the method of Hayman (1954b), it was assumed that the parents were homozygous. Because radish is an allogamous species, the parents used may have a residual heterozygosity at some of the loci controlling shape and size, although they were inbred for several generations. It is theoretically expected that the more highly heterozygous parents will be located farther from the regression line and nearer to the limiting parabola on a $\left(\mathrm{V}_{\mathrm{r}}\right.$, $\mathrm{W}_{\mathrm{r}}$ ) graph (Dickinson and Jinks 1956). In the present study, some parents were located far from the regression line and near the limiting parabola (Fig. 1), indicating the presence of residual heterozygosity in these parents. The application of the method of Hayman (1954b) may, however, be justified, because a simulation study indicated that the existence of heterozygous parents in a diallel table does not influence appreciably the results of analysis if the proportion of heterozygous parents is low (Ukai 2002). It should also be noted that the average degree of dominance could be underestimated if partial heterozygosity remained in the parents (Dickinson and Jinks 1956).

In the diallel analysis according to the method of Hayman (1954b), the absence of epistasis was also assumed. The coefficients of regressions of $\mathrm{W}_{\mathrm{r}}$ on $\mathrm{V}_{\mathrm{r}}$ were not significantly different from unity in the andosol ( $t=0.98$ with $4 \mathrm{df}$, $P=0.19)$ and sandy soil $(t=0.10, P=0.46)$, suggesting that the assumption of the absence of epistasis was verified. In the clayey soil, however, the regression coefficient was significantly different from unity $(t=2.89, P=0.02)$. This suggests that the epistatic effect was present in the inheritance of the bluntness of the distal part (AP2) only in the clayey soil, and

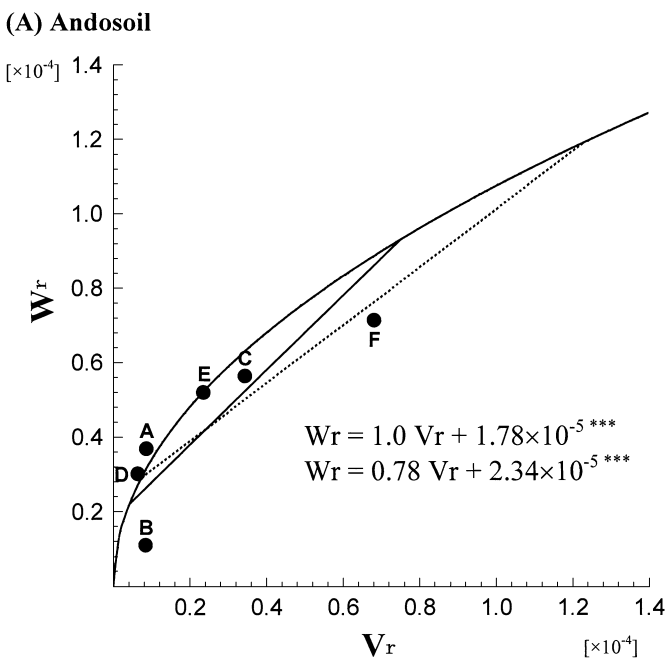

(B) Clayey soil

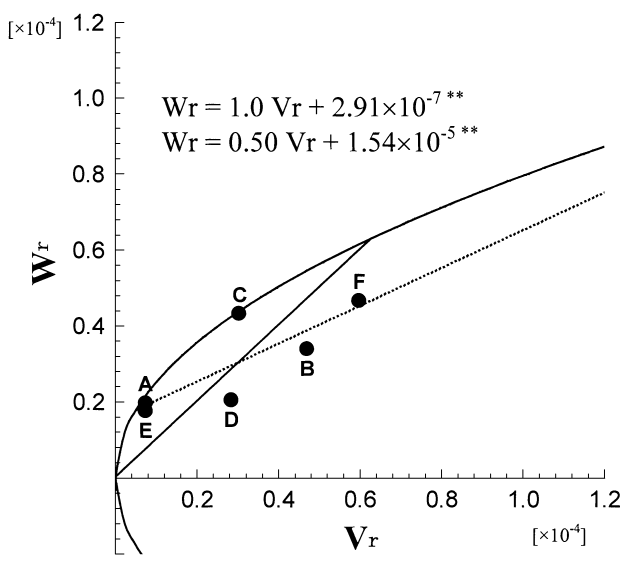

(C) Sandy soil

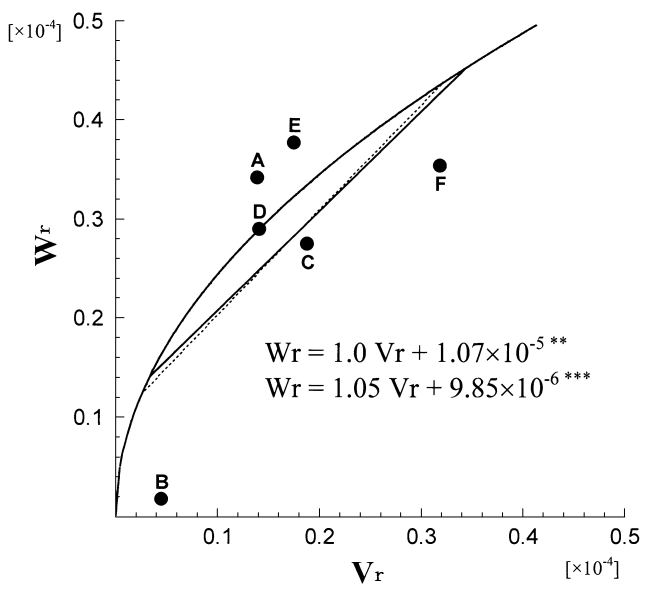

Fig. 1. $\left(V_{r}, W_{r}\right)$ graphs of the 2nd principal component of the group $A$ coefficients (AP2) in three soil types: (A) andosol, (B) clayey soil and (C) sandy soil. The letters A-F indicate the six parental lines. The dotted line represents the best fitting regression of $\mathrm{Wr}$ on $\mathrm{Vr}$ for all the parental lines, and the solid straight line denotes the regression with the theoretical slope of 1 expected in the absence of nonallelic interaction (epistasis). The significance of each regression, which was tested by an analysis of variance, is indicated at the end of the regression equation $(* *$, *** significant at $P<0.01$ or 0.001 , respectively). 
might result in the inaccurate estimation of genetic parameters in the clayey soil unlike in the other soil types (Table 3 ).

The ANOVA of diallel tables showed that the additive effect was significant for all the characteristics. The dominance effects were also significant for all the characteristics, although significant items (i.e., $b_{1}, b_{2}$ or $b_{3}$ ) were different between the characteristics. In contrast, the influence of the soil types was markedly different among the characteristics. The ratio of length to width (AP1) and asymmetric shape variations (BP1 and BP2) were not affected by the differences in soil types. The size characteristics, i.e., two-dimensional projection area (RA) and root weight (RW), and the bluntness of the distal part (AP2) were more affected by the soil types. The interaction between genetic effects and soil type was also significant only for AP2. In this characteristic, the differences in the soil types affected the mode of inheritance (Table 3 and Fig. 1).

In the ANOVA of the diallel table, the interaction between genotype and soil type can be determined by the sum of interactions between genetic effects and soil type (St). In the present study, the item (St) was not significant for all the characteristics of root size and shape. This result was in good agreement with our previous study showing that there was no significant interaction between variety and soil type for most shape characteristics (Iwata et al. 1998). Although the interaction between additive effect (a) and soil type was highly significant for the bluntness of the distal part (AP2), the interaction between genotype and soil type $(\mathrm{St})$ of this characteristic was not significant enough to be detected at the 0.05 or lower level. This indicates that the interaction between the genotype and soil type may not be detected even when some components of the genetic effects (such as the additive effect shown in Table 2) interact with the soil types.

Because the bluntness of the distal part of the root (AP2) is a particularly important characteristic in radish breeding, we determined the cause of the interaction between additive genetic effects and soil type for this characteristic in more detail. Since the component, a, corresponds to the mean deviation from the grand mean due to each parental line, we plotted the mean of progenies of each parent line within each soil type, i.e., $\left(y_{r \cdot s}+y_{\cdot r s}\right) / 2$, where $y_{r \cdot s}$ and $y_{\cdot r s}$ are the means of $r$ th row and $r$ th column of diallel table in soil type $s$, respectively, in order to determine the cause of the interaction (Fig. 2). As a result, parents B, C and D were found to be different from the other parents in the response pattern to the three soil types, indicating that these parents are the main causes of the interaction. This was also indicated by the fact that these parents showed large differences in location on the $\left(\mathrm{V}_{\mathrm{r}}, \mathrm{W}_{\mathrm{r}}\right)$ graphs between the different soil types (Fig. 1).

In the bluntness of the distal part of the root (AP2), the mean effect of parent $\mathrm{E}$ was largest among the parents over different soil types (Fig. 2). Since a blunt-shaped root is preferable to a sharp-shaped one in Japanese markets, parent E may be a useful parent line in crossing if the bluntness should be enhanced. Figure 2 also shows that the mean effect

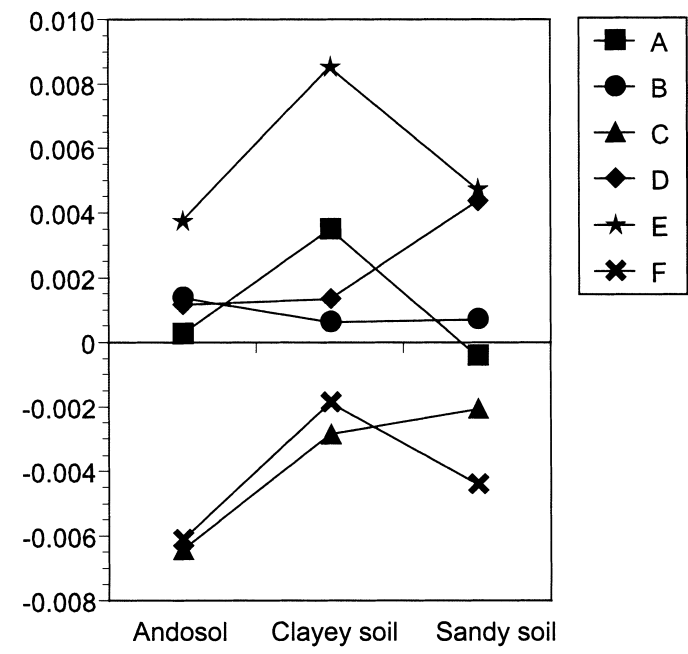

Fig. 2. Means of 2nd principal component of group A coefficients (AP2) for the progenies of parents A to F in different soil types (see text for details).

of parents B and D did not decrease in the andosol and sandy soil (Fig. 2), indicating that these parents may be valuable if the bluntness should be enhanced in these soil types, in which the degree of bluntness of the root generally decreased. Parent B may be useful because of the dominant alleles it harbors (Fig. 1), since the alleles for the blunt-shaped roots were dominant in this characteristic (Table 2).

The results of the present study offer practical suggestions for the improvement of radish cultivars. Because the interaction between genetic effects and soil type does not influence the shape and size characteristics except for the bluntness of the distal part, a desirable genotype that has been selected on a particular soil type can generally be expected to perform similarly in other soil types for these characteristics. In contrast, the interaction between additive effect and soil type was highly significant for the bluntness of the distal part. In the selection of this characteristic, it is necessary to take into account the effect of the soil type and the interaction between genetic effects and soil type. Selection on andosol is recommended, because the heritability was higher in the andosol than in the sandy soil. In the clayey soil, the effect of epistasis should be taken into account in the selection.

\section{Acknowledgements}

We are grateful to Dr. Ryo Ohsawa (Institute of Agriculture and Forestry, University of Tsukuba) and Dr. Arthur Villordon (Sweet Potato Research Station, Louisiana State University Agricultural Center) for critically reading this manuscript and providing useful suggestions.

\section{Literature Cited}

Dickinson,A.G. and J.L.Jinks (1956) A generalized analysis of diallel analysis. Genetics 41: 65-78. 
Goldman,I.L. (1995) Differential effect of population density on shape and size of cylindrical red beet (Beta vulgaris L.) genotypes. J. Amer. Soc. Hort. Sci. 120: 906-908.

Hayman, B.I. (1954a) The analysis of variance of diallel tables. Biometrics 10: 235-244.

Hayman, B.I. (1954b) The theory and analysis of diallel crosses. Genetics 39: 789-809.

Iwata,H., S.Niikura, S.Matsuura, Y.Takano and Y.Ukai (1998) Evaluation of variation of root shape of Japanese radish (Raphanus sativus L.) based on image analysis using elliptic Fourier descriptors. Euphytica 102: 143-149.

Iwata,H., S.Niikura, S.Matsuura, Y.Takano and Y.Ukai (2000) Diallel analysis of root shape of Japanese radish (Raphanus sativus L.) based on elliptic Fourier descriptors. Breed. Sci. 50: 73-80.

Iwata,H. and Y.Ukai (2002) SHAPE: A computer program package for quantitative evaluation of biological shapes based on elliptic Fourier descriptors. J. Hered. 93: 384-385.

Kuhl,F.P. and C.R.Giardina (1982) Elliptic Fourier features of a closed contour. Computer Graphics and Image Processing 18: 236-258.

Mather,K. and J.L.Jinks (1971) Biometrical Genetics. Chapman and Hall, London. 382 p.

Niikura, S. and S.Matsuura (1999) Genetic variation of the $S$-alleles and level of self-incompatibility in Japanese cultivated radish
(Raphanus sativus L.). Breed. Res. 1: 211-220 (in Japanese with English summary).

Nishiyama,I. (1958) Nihon No Daikon. Nihon Gakujutsu Sinkokai, Tokyo. $161 \mathrm{p}$ (in Japanese).

Rohlf,F.J. and J.W.Archie (1984) A comparison of Fourier methods for the description of wing shape in mosquitoes (Ritera culicidae). Syst. Zool. 33: 302-317.

Rosenfeld,H.J., R.T.Samuelsen and P.Lea (1998a) The effect of temperature on sensory quality, chemical composition and growth of carrot (Daucus carota L.) I. Constant diurnal temperature. J. Hort. Sci. Biotech. 73: 275-288.

Rosenfeld,H.J., R.T.Samuelsen and P.Lea (1998b) The effect of temperature on sensory quality, chemical composition and growth of carrot (Daucus carota L.). II. Constant diurnal temperatures under different seasonal light regimes. J. Hort. Sci. Biotech. 73: 578-588.

Rosenfeld,H.J., R.T.Samuelsen and P.Lea (1999) The effect of temperature on sensory quality, chemical composition and growth of carrot (Daucus carota L.). III. Different diurnal temperature amplitudes. J. Hort. Sci. Biotech. 74: 196-202.

Ukai,Y. (2002) Genetic analysis of quantitative traits. Igaku Shuppan, Tokyo. $199 \mathrm{p}$ (in Japanese).

Villordon,A. and H.Carroll (2002) Digital image analysis of sweetpotato storage roots in herbicide trials. HortScience 37: 669-670. 